\title{
Influence of Gaussian and Saw-tooth pulses in modulating excitation kinetics of impurity doped quantum dots
}

\author{
Suvajit $\mathrm{Pal}^{1}$ and Manas Ghosh ${ }^{2 *}$ \\ ${ }^{1}$ Department of Chemistry, Hetampur Raj High School, Hetampur, Birbhum 731124, West Bengal, India. \\ * 2 Department of Chemistry, Physical Chemistry Section, Visva-Bharati University, Santiniketan, Birbhum \\ 731235, West Bengal, India.
}

Tel: 91-3463-261526, Email: pcmg77@rediffmail.com

doi:10.5618/chem.2012.v2.n1.7 || Received: 03-11-2012, Accepted: 28-12-2012, Available online: 03-01-2013

\begin{abstract}
We explore the excitation kinetics in a single electron generic quantum dot theoretically. The quantum dot is doped with a repulsive impurity and subject to a pulsed field of Gaussian and saw-tooth pulse shapes that induce the excitation. The impurity potential has been assumed to have a Gaussian nature. The investigation reveals the sensitivity of the typical shape of the pulse, alongside the influences of dopant location, number of pulse, and some other characteristic pulse parameters towards modulating the excitation rate. Specifically, the other characteristic pulse parameters involve the width of the Gaussian pulse and the height of the saw-tooth pulse. In all the cases the typical pulse shapes announce its role on excitation kinetics unhesitatingly through a number of observations. The present study has also indicated enough evidence of twist in the mutual dominance of several factors that could favor and impede excitation accompanying the shift of dopant location. Importantly, those factors also depend severely on the pulse shape. The pulse shape and its characteristic parameters interfere delicately with the interplay between impurity location and number of pulses fed into the system and emerge as an important ingredient that can engineer the excitation kinetics.
\end{abstract}

Keywords: quantum dot; impurity doping; dopant coordinate; pulsed field; Gaussian pulse shape; sawtooth pulse shape; excitation rate.

\section{Introduction}

Over the couple of decades impurity doping in semiconductor materials has emerged as a useful technology that has been utilized to control optoelectronic properties of a wide range of semiconductor devices [1, 2]. Impurity doping in quantum dots (QD) invariably invites new perspectives and subtleties in the field of applied physics. This happens because of the interplay between various confinement sources with impurity potentials [3]. Moreover, the dopant location shows some crucial role in the said interplay and profoundly alters the electronic and optical properties of the system [4-8]. Naturally, we find a vast literature comprising of theoretical studies of impurity states [911] particularly emphasizing the role of dopant location $[12,13]$. Investigations on fabricating the impurity states have received further impetus with the development of sophisticated experimental techniques such as molecular beam epitaxy, liquid phase epitaxy, and chemical vapor deposition. Propelled by this, there are also some excellent experimental works which encompass the mechanism and control of dopant incorporation [14, 15].

The emergence of novel experimental and theoretical techniques along with the existing ones has encouraged the research on carrier dynamics in nanodevices. The time-dependent aspects in nanodevices naturally become a hotly pursued research topic. The internal transitions between impurity induced states in a QD led to research on carrier dynamics $[16,17]$. These transitions depend on the spatial restriction imposed by the impurity. A close scrutiny of the above dynamical features suggests us to explore the excitation of electrons strongly confined in QDs. Analysis on this aspect deems importance since it offers us model systems for use in opto-electronic devices and as lasers. Within the purview of engineering applications excitation in QD assumes importance in optical encoding, multiplexing, photovoltaic and light emitting devices. The phenomenon also plays some notable role in the eventual population transfer among the exciton states in QD $[18,19]$.

Recently, we have made some investigations on the excitation profiles of the doped quantum dots initiated 
by discontinuously reversing pulsed field [20, 21]. In this paper we have attempted to investigate the excitation in the doped quantum dot exposed to a pulsed field which is continuous in nature. The motivation for the work originates from a survey of recent works which study the effect of applied electric field on doped quantum wells and dots [22-24]. Also there is another work which involves pulsed electromagnetic field in a double QD structure [25]. Looking at a more elaborate investigation of the pulsed field we have exploited pulses of Gaussian and Saw-tooth shapes to ascertain if a variation in the shape can modulate the target excitations. A variation in the pulse shape basically implies a change in the pattern of energy transfer from the external field to the QD. It seems quite interesting to decipher how such a change in the mode of field-to-dot energy transfer affects the excitation kinetics. The present investigation assumes that the QD is doped with a repulsive Gaussian impurity which simulates dopant with excess electrons [26, 27]. In the present work the time-dependence has been modeled by attaching a potential $V(t)$ to the dot Hamiltonian. A consequent follow up of the dynamics of the doped dot in the timedependent potential becomes the obvious next task to handle the problem. Alongside pulse shape, we have also analyzed the roles played by the number of pulses fed into the system from the external field $\left(n_{p}\right)$ as well as the dopant location $\left(r_{0}\right)$ on excitation kinetics. Moreover, the width of the Gaussian pulse and the height of the saw-tooth pulse have also been found to modulate the excitation kinetics. We have attempted to rationalize thoroughly the influences of above factors as well as the delicate interplay among them on the excitation profile in the present enquiry.

\section{Method}

The model considers an electron subject to a harmonic confinement potential $V(x, y)$ and a perpendicular magnetic field $B$. The confinement potential assumes the form $V(x, y)=(1 / 2) m * \omega_{0}^{2}\left(x^{2}+y^{2}\right)$, where $\omega_{0}$ is the harmonic confinement frequency, $\omega_{c}=\frac{e B}{m^{*} c}$ being the cyclotron frequency (a measure of magnetic confinement offered by $B$ ). In the present work a magnetic field of miliTesla (mT) order has been employed. $m^{*}$ is the effective electronic mass within the lattice of the material to be used. We have taken $m^{*}$ $=0.5 m_{0}$ and set $\hbar=\mathrm{e}=m_{0}=\mathrm{a}_{0}=1$. This value of $m^{*}$ closely resembles $G e$ quantum dots ( $m^{*}=0.55$ a.u.). We have used Landau gauge $[A=(B y, 0,0)]$ where A stands for the vector potential. The Hamiltonian in our problem reads

$$
\begin{aligned}
& H_{0}^{\prime}=-\frac{\hbar^{2}}{2 m^{*}}\left(\frac{\partial^{2}}{\partial x^{2}}+\frac{\partial^{2}}{\partial y^{2}}\right)+\frac{1}{2} m^{*} \omega_{0}^{2} x^{2}+\frac{1}{2} m *\left(\omega_{0}^{2}+\omega_{c}^{2}\right) y^{2} \\
& -i \hbar \omega_{c} y \frac{\partial}{\partial x}
\end{aligned}
$$

Define $\Omega^{2}=\omega_{0}^{2}+\omega_{c}^{2}$ as the effective frequency in the y-direction. The model Hamiltonian [cf. eqn(1)] sensibly represents a 2-d quantum dot with a single carrier electron [28, 29]. The form of the confinement potential conforms to kind of lateral electrostatic confinement of the electrons in the $x-y$ plane. In real QDs the electrons are confined in 3-dimensions i.e. the carriers effectively possess a quasi-zero dimensional domain. The confinement length scales $R^{l}, R^{2}$, and $R^{3}$ can, in general, be different in three spatial directions, but usually $R^{3} \ll R^{l} \simeq R^{2}$. Whenever such QDs are modeled $R^{3}$ is often taken to be strictly zero and the confinement in the other two directions is described by a potential $V$ with $V(x) \rightarrow \infty$ for $|x| \rightarrow \infty, x=\left(x^{1}, x^{2}\right) \in R^{2}$. More often than not, a parabolic confinement potential $\left(V=\frac{1}{2} \omega|x|^{2}\right)$ is chosen as it appears quite realistic and largely reduces computational cost [7-9, 13, 22]. Assuming that the z-extension could be effectively considered zero, the electronic properties in these nanostructures have been successfully described using the model of 1 - electron motion in 2-d harmonic oscillator potential in the presence of a magnetic field $[28,29]$.

Now, as the impurity perturbation is attached to the Hamiltonian [cf. eqn(1)] it transforms to

$H_{0}\left(x, y, \omega_{\mathrm{c},} \omega_{0}\right)=H_{0}^{\prime}\left(x, y, \omega_{\mathrm{c}}, \omega_{0}\right)+V_{\text {imp }}\left(x_{0}, y_{0}\right)$,

where $V_{\text {imp }}\left(x_{0}, y_{0}\right)=V_{\text {imp }}(0)=V_{0} e^{-\gamma\left[\left(x-x_{0}\right)^{2}+\left(y-y_{0}\right)^{2}\right]}$ with $\gamma>0$ and $V_{0}>0$ for repulsive impurity, and $\left(x_{0}\right.$, $y_{0}$ ) denotes the coordinate of the impurity center. $\mathrm{V}_{0}$ is a measure of the strength of impurity potential whereas 
$\gamma^{-1}$ determines the spatial stretch of the impurity potential. A large value of $\gamma$ indicates a highly quenched spatial extension of impurity potential whereas a small $\gamma$ accounts for spatially dispersed one. The parameter $\gamma$ in the impurity potential is equivalent to $\frac{1}{d^{2}}$, where $d$ is proportional to the width of the impurity potential $[26,27]$. The value of $\gamma$ is taken to be 0.001 a.u. which corresponds to an extension of the impurity domain up to $1.41 \mathrm{~nm}$. The dopant strength $\left(V_{0}\right)$ assumes a maximum value of $\sim 10^{-4}$ a.u. or 2.72 meV. The use of such Gaussian impurity potential is quite well-known [30-32].

We write the trial wave function $\psi(x, y)$ as a superposition of the product of harmonic oscillator eigenfunctions $\emptyset_{n}(\alpha x)$ and $\emptyset_{m}(\beta y)$ respectively as follows $[20,21]$ :

$$
\psi(x, y)=\sum_{n, m} C_{n, m} \emptyset_{n}(\alpha x) \emptyset_{m}(\beta y),
$$

where $C_{n, m}$ are the variational parameters and $\alpha=$ $\sqrt{\frac{m^{*} \omega_{0}}{\hbar}}$ and $\beta=\sqrt{\frac{m^{*} \Omega}{\hbar}}$. In the linear variational calculation, we have used an appreciably large number of basis functions [cf. eqn(3)] with $n, m=0-20$ for each of the directions $(x, y)$. This direct product basis spans a space of $(21 \times 21)$ dimension. It has been verified that the basis of such size scans the $2-\mathrm{d}$ space effectively completely as long as monitoring the observables under investigation is concerned. A convergence test run by us with still greater number of basis functions confirmed our observation.

The general expression for the matrix elements of $H_{0}^{\prime}$ in the chosen basis are as follows:

$$
\begin{aligned}
& \left(H_{0}^{\prime}\right)_{n, m ; n^{\prime} m^{\prime}}=\hbar\left\{\left(n^{\prime}+\frac{1}{2}\right) \omega_{0}+\left(m^{\prime}+\frac{1}{2}\right) \sqrt{\omega_{0}^{2}+\omega_{c}^{2}}\right\} \delta_{n, n^{\prime}} \delta_{m, m^{\prime}} \\
& -i \hbar \omega_{c}^{\frac{\alpha}{\beta}} \\
& {\left[\left\{\sqrt{\frac{n^{\prime}}{2}} \delta_{n^{\prime}-1, n}-\sqrt{\frac{n^{\prime}+1}{2}} \delta_{n^{\prime}+1, n}\right\}\left\{\sqrt{\frac{m^{\prime}+1}{2}} \delta_{m^{\prime}+1, m}+\sqrt{\frac{m^{\prime}}{2}} \delta_{m^{\prime}-1, m}\right\}\right] .}
\end{aligned}
$$

And the matrix elements of $V_{\text {imp }}$ are given by [20]

$$
\begin{aligned}
& \left(V_{i m p}^{\prime}\right)_{n, m ; n^{\prime}, m^{\prime}}= \\
& {\left[V_{0}\left(D_{1} D_{2}\right)\right] \sum_{k=0}^{\min \left(n, n^{\prime}\right)} \sum_{l=0}^{\min \left(m, m^{\prime}\right)} f\left(k, n, n^{\prime}\right) g\left(l, m, m^{\prime}\right),}
\end{aligned}
$$

Where,

$f\left(k, n, n^{\prime}\right)=2^{k} k !{ }_{k}^{n} C_{k}^{n^{\prime}} C\left(1-\alpha^{* 2}\right)^{\frac{n+n^{\prime}}{2}-k} H_{n+n^{\prime}-2 k}\left(\alpha_{1} \rho_{1}\right)$,

and

$g\left(l, m, m^{\prime}\right)=$

$2^{l} l !{ }_{l}^{m} C^{m_{l}^{\prime}} C\left(1-\beta^{* 2}\right)^{\frac{m+m^{\prime}}{2}-l} H_{m+m^{\prime}-2 l}\left(\beta_{1} \rho_{2}\right)$.

The other relevant quantities are

$$
\begin{aligned}
& D_{1}=\frac{A \lambda_{1} \pi^{\frac{1}{2}}}{\delta_{1}}, D_{2}=\frac{B \lambda_{2} \pi^{\frac{1}{2}}}{\delta_{2}}, A=\frac{\alpha}{\left(2^{n+n^{\prime}} n ! n^{\prime} ! \pi\right)^{\frac{1}{2}}} \\
& B=\frac{\beta}{\left(2^{m+m^{\prime}} m ! m^{\prime} ! \pi\right)^{\frac{1}{2}}}, \delta_{1}^{2}=\alpha^{2}+\gamma, \delta_{2}^{2}=\beta^{2}+ \\
& \gamma, \lambda_{1}=\exp \left[-\frac{\gamma x_{0}^{2}\left(\delta_{1}^{2}-\gamma\right)}{\delta_{1}^{2}}\right], \text { and } \lambda_{2}= \\
& \exp \left[-\frac{\gamma y_{0}^{2}\left(\delta_{2}^{2}-\gamma\right)}{\delta_{2}^{2}}\right], \rho_{1}=\frac{\gamma x_{0}}{\delta_{1}}, \rho_{2}=\frac{\gamma y_{0}}{\delta_{2}}, \alpha^{*}=\frac{\alpha}{\delta_{1}}, \\
& \beta^{*}=\frac{\beta}{\delta_{2}}
\end{aligned}
$$

And $H_{n}(x)$ stands for the Hermite polynomials of $n^{\text {th }}$ order.

The $p^{\text {th }}$ eigenstate of the system in this representation can be written as

$$
\psi_{\mathrm{p}}(x, y)=\sum_{i, j} C_{i j, p}\left\{\phi_{i}(\alpha x) \phi_{j}(\beta y)\right\}
$$

where $i, j$ are the appropriate quantum numbers, respectively and $(i j)$ are composite indices specifying the direct product basis. The external pulsed electric field $\varepsilon(t)$ is now switched on with $\varepsilon_{x}(t)=$ $\varepsilon_{x}(0) S(t) \sin \left(v_{x} t\right)$ for $x$-direction, and analogously,

$\varepsilon_{y}(t)=\varepsilon_{y}(0) S(t) \sin \left(v_{y} t\right)$ for $y$-direction. $\varepsilon_{x}(0)$ and $\varepsilon_{y}(0)$ are the field intensities along $x$ and $y$ directions at $t=0$ and $v_{x}$ and $v_{y}$ being the oscillation frequencies in $x$ and $y$ directions, respectively. Generally speaking, the pulsed field $\varepsilon(\mathrm{t})$ has been considered to be characterized by intensity modulated by a pulse-shape function $S(t)$ where, 


$$
\varepsilon(t)=\varepsilon(0) S(t) \sin (v t)
$$

The pulse has peak field strength $\varepsilon(0)$, and a fixed frequency $v$. We have considered two different pulse shape functions, namely, Gaussian and saw-tooth which assume the following analytical expressions:

$S(t)=\exp \left[-k\left(t-T_{p}\right)^{2}\right], \quad$ Gaussian pulse

$S(t)=a\left(1-\frac{t}{T_{p}}\right), \quad$ Saw-tooth pulse.

where $T_{p}$ stands for pulse duration time. Thus $T_{p}$, or equivalently $n_{p}$ (the number of pulses), appears to be a key control parameter. Figs. 1(a)-(b) depict the profiles of five consecutive pulses of above two shapes as a function of time for a ready comparison. For better visualization we also provide the full intensity profile $\varepsilon(t)$ as a function of time for Gaussian and Saw-tooth pulses [figs. 1(c) and 1(d)]. As the pulsed field begins interacting with the dot electron, the time-dependent part of the Hamiltonian reads

$$
V(t)=\varepsilon_{x}(t) x|e|+\varepsilon_{y}(t) y|e|
$$

Now the time-dependent Hamiltonian reads

$$
H(t)=H_{0}+V(t)
$$

The matrix element involving any two arbitrary eigenstates $p$ and $q$ of $H_{0}$ due to $V(t)$ reads

$\left.V(p, q)=\left\langle\psi_{p}|V(t)| \psi_{q}\right\rangle\right\rangle=\varepsilon_{x}(0) S(t) \sin \left(v_{x} t\right)$

$\sum_{i j} \sum_{k l} C_{i j, p}^{*} C_{k l, q}\left\langle\phi_{i}(\alpha x)|x| \phi_{k}(\alpha x)\right\rangle \delta_{j l}+$

$\varepsilon_{y}(0) S(t) \sin \left(v_{y} t\right) \sum_{i j} \sum_{k l} C_{i j, p}^{*} C_{k l, q}\left\langle\phi_{j}(\beta y)|y| \phi_{l}(\beta y)\right\rangle \delta_{i k}$, (10)

where

$\left\langle\phi_{i}(\alpha x)|x| \phi_{k}(\alpha x)\right\rangle=\frac{1}{\alpha}\left[\sqrt{\frac{k+1}{2}} \delta_{k, i}+\sqrt{\frac{k}{2}} \delta_{k-1, i}\right]$,

and

$\left\langle\phi_{j}(\beta y)|y| \phi_{l}(\beta y)\right\rangle=\frac{1}{\beta}\left[\sqrt{\frac{l+1}{2}} \delta_{l, j}+\sqrt{\frac{l}{2}} \delta_{l-1, j}\right]$.

$H_{0}$ is diagonal in the $\{\psi\}$ basis. Under the external perturbation, the evolving wave function is described by a linear combination of the eigenstates of $H_{0}$.

$\psi(x, y, t)=\sum_{q} a_{q}(t) \psi_{q}$,

In order to determine the time-dependent superposition coefficients we need to solve the timedependent Schrödinger equation (TDSE).

$$
i \hbar \frac{\partial \psi}{\partial t}=H \psi \quad \text { or equivalently }
$$

$$
i \hbar a_{q}(t)=H a_{q}(t)
$$

with the initial conditions $a_{p}(0)=1, a_{q}(0)=0$, for all $q$ $\neq p$, where $p$ may be the ground or any other excited states of $H_{0}$. The TDSE in the direct product basis [cf. eqn(14)] has been integrated by the 6-th order RungeKutta-Fehlberg method with a time step size $\Delta t=0.01$ a.u. and the numerical stability of the integrator has been checked. The quantity $P_{k}(t)=\left|a_{k}(t)\right|^{2}$ indicates the population of $k^{\text {th }}$ state of $H_{0}$ at time $t$. There occurs a continuous growth and decay in the ground state population $\left[P_{0}(t)\right]$ during the time evolution. Naturally, the quantity $Q(t)=1-P_{0}(t)$ serves as a measure of excitation. In consequence, the quantity $R_{e x}(t)=\frac{d Q}{d t}$ serves as the time-dependent rate of excitation. We have calculated the time-average rate of excitation $\left[\left\langle R_{e x}(t)\right\rangle=\frac{1}{T} \int_{0}^{T} R_{e x}(t) d t\right]$ with $T$ being the total time of dynamic evolution $(\sim 100$ ps in the present investigation). The dopant location, the numbers of pulses fed into the system, a few pulse parameters, and obviously the particular shape of the pulse have been found to influence this rate in a coupled manner and shed light on some important features of excitation profile.

\section{Results and Discussion}

Let us now start our discussions on the finer details of the dynamical aspects. We are already preoccupied with the anticipation that the dopant location $\left(r_{0}\right)$ could interplay with $n_{p}$ subtly to influence the excitation profile. The anticipation originates from a survey of the notable works $[3,6,12]$ related to dopant located at offcenter position which are worth mentioning. Figs. 1(a) and (b) exhibit the actual shape of the Gaussian and Saw-tooth pulses, respectively, where the pulse shape function $(S(t))$ is plotted against $(t)$ for five successive pulses. The exact shape of the external pulsed electric field $(\varepsilon(t))$ is unveiled at Figs. 1(c) and (d) where $(\varepsilon(t))$ is plotted against $(t)$ for Gaussian and Saw-tooth pulses, respectively. Figs. 2(a) and (b) display the instantaneous excitation $[Q(t)]$ as a function of time for Gaussian and saw-tooth pulse shapes, respectively. In these plots we consider on-center $\left(r_{0}=0.0\right.$ a.u. $)$, near off-center $\left(r_{0}=\right.$ 28.28 a.u.), and far off-center ( $r_{0}=70.71$ a.u.) dopant locations as representative cases. In all these plots the number of pulse has been kept equal to 7. A close look at these plots does not reveal any significant role played by the dopant coordinate so far as instantaneous excitation is concerned. In all the three dopant coordinates, more or less, the Gaussian pulse shows 
abrupt jumps and falls, whereas the saw-tooth pulses, after an initial rise, continue to make highly irregular appearances. Thus, we feel that, it needs somewhat more sincere inspection in order to realize the role of dopant location on excitation kinetics comprehensively.

Table 1: The details of pulse shape function $S(t)$ along with $n_{p}$ values corresponding to minimization $\left(n_{p, \min }\right)$, saturation $\left(n_{p, s a t}\right)$, and depth of minima.

On-center dopant

\begin{tabular}{|lccc|}
\hline$S(t)$ & $n_{p, \min }$ & $n_{p, \text { sat }}$ & Depth of minima \\
\hline Gaussian & 7 & 12 & Moderate \\
Saw-tooth & 8 & 13 & Large \\
\hline
\end{tabular}

Near off-center dopant

\begin{tabular}{|lccc|}
\hline$S(t)$ & $n_{p, \min }$ & $n_{p, \text { sat }}$ & Depth of minima \\
\hline Gaussian & 8 & 12 & Large \\
Saw-tooth & 7 & 10 & Moderate \\
\hline
\end{tabular}

Far off-center dopant

\begin{tabular}{|lccc|}
\hline$S(t)$ & $n_{p, \min }$ & $n_{p, \text { sat }}$ & Depth of minima \\
\hline Gaussian & - & 6 & - \\
Saw-tooth & 10 & 14 & Moderate \\
\hline
\end{tabular}
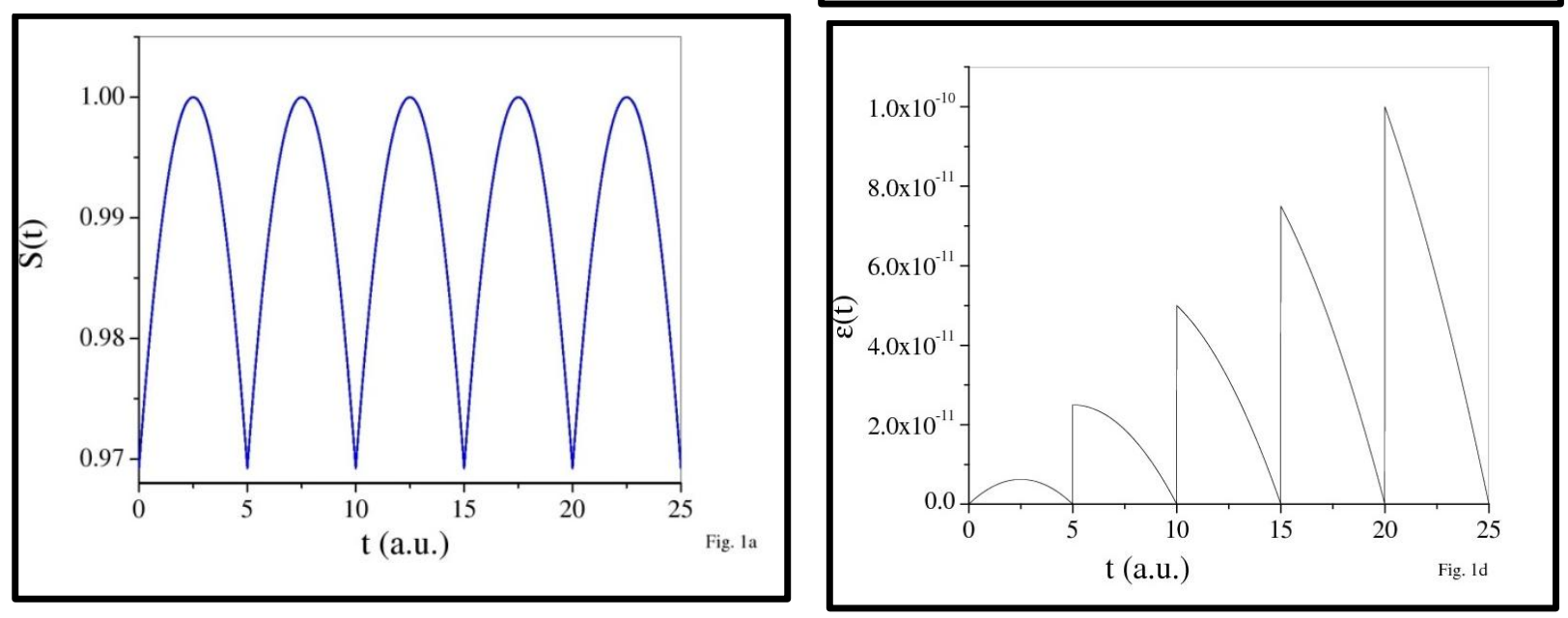

Fig. 1. Plot of pulse shape function $S(t)$ against $t$ for five
successive pulses: (a) Gaussian pulse and (b) Saw-tooth
pulse. Plot of external pulsed electric field $\varepsilon(t)$ against $t$ :
(c) Gaussian pulse and (d) Saw-tooth pulse.

Fig. 1. Plot of pulse shape function $S(t)$ against $t$ for five
successive pulses: (a) Gaussian pulse and (b) Saw-tooth
pulse. Plot of external pulsed electric field $\varepsilon(t)$ against $t$ :
(c) Gaussian pulse and (d) Saw-tooth pulse.

Fig. 1. Plot of pulse shape function $S(t)$ against $t$ for five
successive pulses: (a) Gaussian pulse and (b) Saw-tooth
pulse. Plot of external pulsed electric field $\varepsilon(t)$ against $t$ :
(c) Gaussian pulse and (d) Saw-tooth pulse.

Fig. 1. Plot of pulse shape function $S(t)$ against $t$ for five
successive pulses: (a) Gaussian pulse and (b) Saw-tooth
pulse. Plot of external pulsed electric field $\varepsilon(t)$ against $t$ :
(c) Gaussian pulse and (d) Saw-tooth pulse.
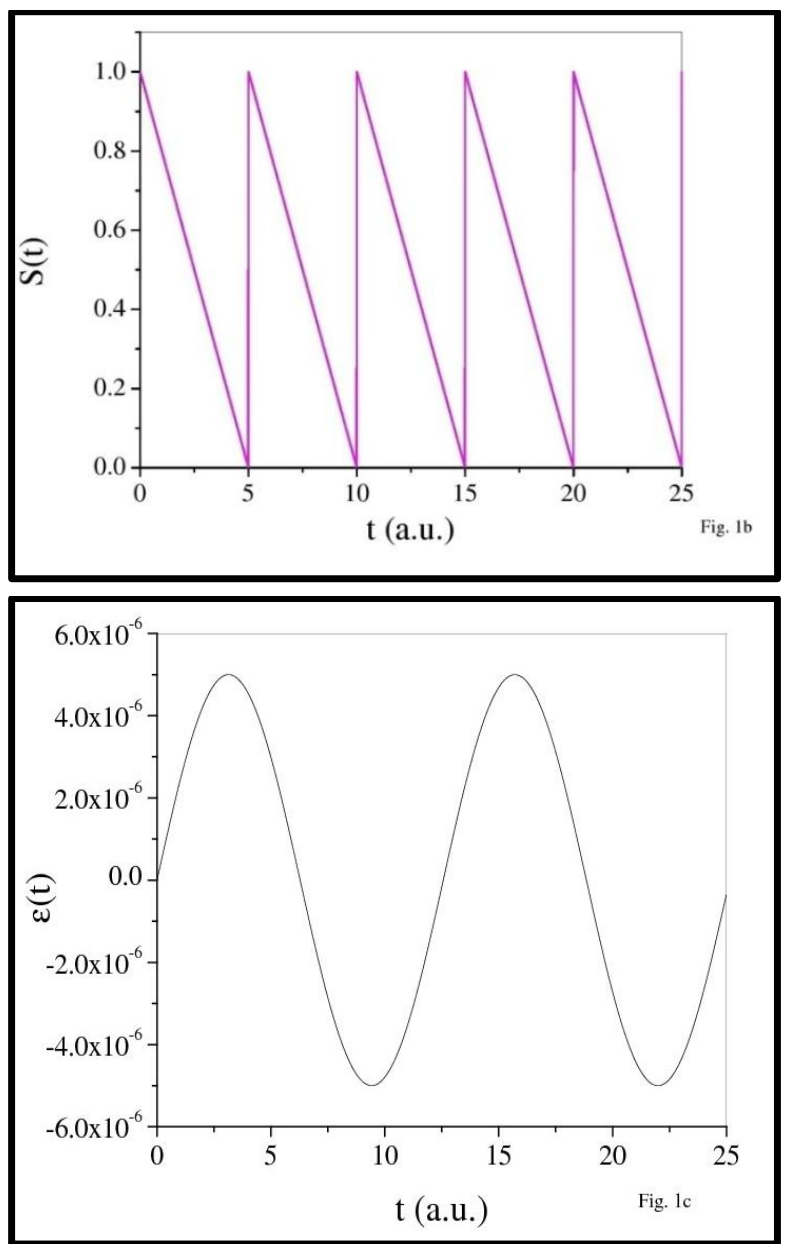


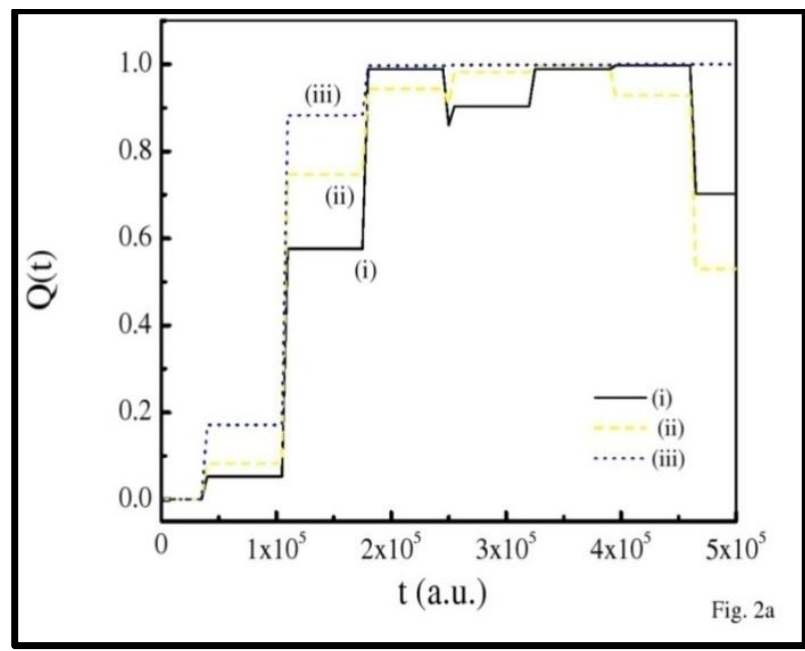

Fig. 2a. Plot of $Q(t)$ against $\mathrm{t}$ with $V_{0}=1.0 \times 10^{-6}$ a.u., $\gamma$ $=0.001$ a.u., and $n_{p}=7$ for Gaussian pulse for different dopant locations: (i) on-center ( $r_{0}=0.0$ a.u.), (ii) near off-center $\left(r_{0}=28.28\right.$ a.u. $)$, and (iii) far off- center $\left(r_{0}=\right.$ 70.71 a.u.).

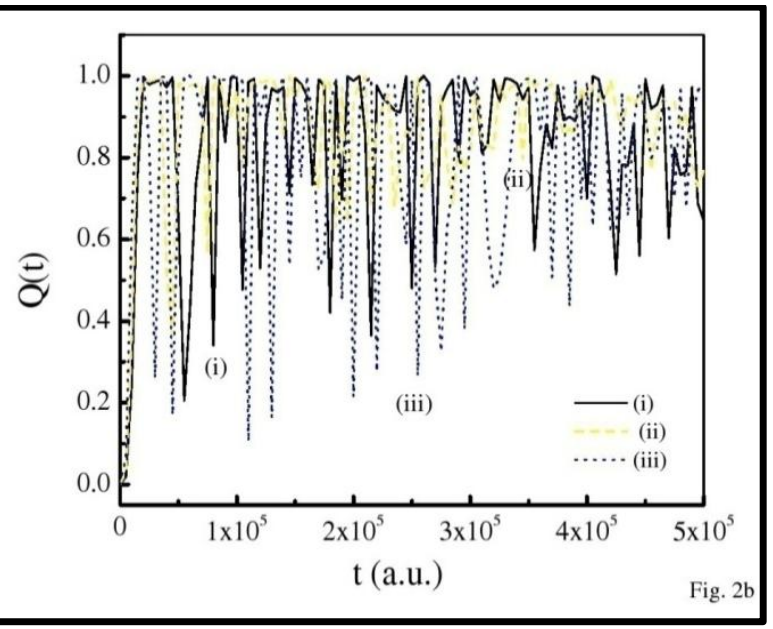

Fig. 2b. Plot of $Q(t)$ against $t$ with $V_{0}=1.0 \times 10^{-6}$ a.u., $\gamma$ $=0.001$ a.u., and $n_{p}=7$ for saw-tooth pulse for different dopant locations: (i) on-center $\left(r_{0}=0.0\right.$ a.u.), (ii) near off-center $\left(r_{0}=28.28\right.$ a.u.), and (iii) far off-center $\left(r_{0}=\right.$ 70.71 a.u.).

In view of this, however, simple monitoring of instantaneous excitation turns out to be inadequate and it needs a more critical analysis. Thus, we realize that it is necessary to determine the time-average excitation rate $\left(\left\langle R_{e x}\right\rangle\right)$ to assess the role of dopant location or more importantly, the nature of interplay between the dopant position and the pulse number. As the dopant is introduced at a greater distance from the dot confinement center $\left(r_{0}=0\right)$ the confines of electric $\left(\omega_{0}\right)$ and magnetic $\left(\omega_{c}\right)$ origins naturally become weak and favor excitation. In order to visualize the role played by the spatial stretch of impurity potential we have plotted the matrix elements $\left\langle\psi_{0}\left|\widehat{V}_{i m p}\right| \psi_{0}\right\rangle$ as a function of radial position of impurity $\left(r_{0}\right)$ [Fig. 3]. The plot nicely delineates the overlap of the impurity potential with the ground state wave function $\left|\psi_{0}\right\rangle$. As expected, the plot reveals a continually decreasing dot-impurity overlap with gradual shift of the dopant away from the dot confinement center. However, at large $r_{0}$ the overlap settles to an otherwise steady value. Thus, quite naturally, the excitation process is influenced by a variation of dopant location.

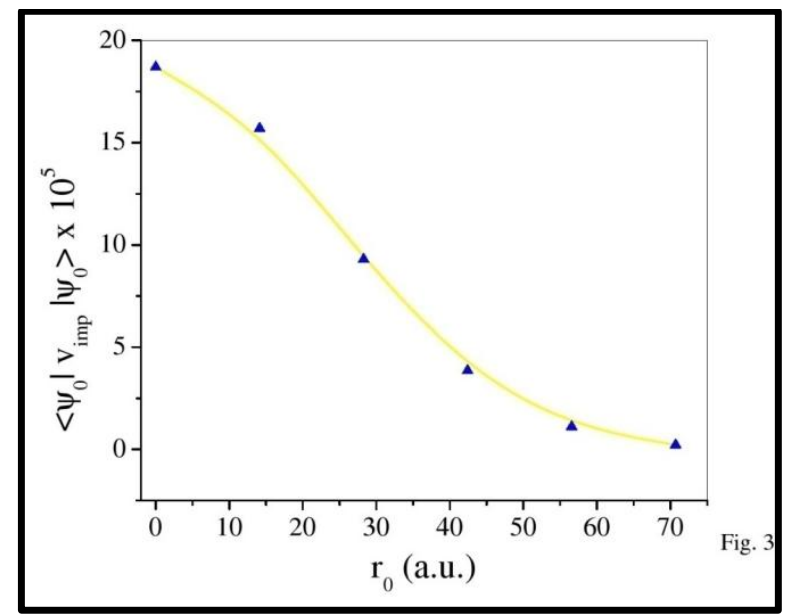

Fig. 3. Plot of $\left\langle\psi_{0}\left|\hat{V}_{i m p}\right| \psi_{0}\right\rangle$ against $r_{0}$ with $V_{0}=1.0$ $\times 10^{-6}$ a.u. and $\gamma=0.001$ a.u.

Let us now have a close look at the plot that delineates the time-average excitation rate $\left(\left\langle R_{e x}\right\rangle\right)$ as a function of $\left(n_{p}\right)$ for three different dopant locations; oncenter $\left(r_{0}=0.0\right.$ a.u. $)$, near off-center $\left(r_{0}=28.28\right.$ a.u. $)$, and far off-center $\left(r_{0}=70.71\right.$ a.u.) [Figs. 4(a-b)]. Figs. 4a and $4 \mathbf{b}$ depict the related plots for Gaussian and sawtooth pulses, respectively. In these plots we have varied $n_{p}$ from 1 to 20 . This range of $n_{p}$ is expected to reveal the influence of pulse on the extent of excitation. The plots clearly evince the dependence of excitation rate on number of pulse mingled with diversities arising out of dopant location. For an on-center dopant, $\left(\left\langle R_{e x}\right\rangle\right)$ has been found to pass through a minima for both the pulse shapes, however, the location as well as the depth of the minima depends on the shape. The minimization in the excitation rate takes place at around $n_{p} \approx 7$ and $n_{p} \approx 8$ for Gaussian and saw-tooth pulses, respectively. Also, whereas the minimum is manifestly deep for saw-tooth pulses, it is quite moderate for the Gaussian pulse. Moreover, for both the pulse shapes, we envisage saturation in the excitation rate beyond $n_{p} \approx 10$ 
signifying kind of compromise between the influences that favor and oppose the excitation process. The specific values of $n_{p} \mathrm{~s}$ beyond which the saturation begins also depend on the nature of pulse shape and read approximately 12 and 13 for Gaussian and saw-tooth pulses, respectively. In the small $n_{p}$ domain, an increase in $n_{p}$ supplies more and more energy to the system to a very limited extent. Since the strong confinement resists any attempt to promote excitation, it does so more enthusiastically as $n_{p}$ increases up to a small value forcing the excitation rate to a minimum. After the minima the large amount of energy input outweighs the confinement strength and the rate begins to rise. At very large $n_{p}$, the excitation process gets accompanied by considerable deexcitation and a compromise between them leads to saturation. Overall, from the above discussions it comes out that in the on-center dopant location, the pulse shape indeed marks its signature on the excitation kinetics. By and large, the excitation kinetics follows more or less similar pattern with variation of $n_{p}$ depending on the factors that foment and impede excitation for both the pulse shapes. However, the nuances of Gaussian and saw-tooth shapes on excitation kinetics are exhibited via the subtle variations in the $n_{p}$ values corresponding to location of minima, the onset of saturation and also examining the depth of minima.

At near off-center dopant location ( $r_{0}=28.28$ a.u.) the similar plots carry some interesting features. Although for both the pulse shapes we again observe minimization in $\left\langle R_{e x}\right\rangle$ followed by saturation as a function of $n_{p}$ as before, but the extent of minimization has been severely depleted for the saw-tooth pulse. On the other hand, for the Gaussian shaped pulse the minimum appears to be deeper now. The locations of minimization being $n_{p} \approx 8$, and $n_{p} \approx 7$ for Gaussian and saw-tooth pulses, respectively. The saturation $n_{p}$ values now become approximately 12 and 10 for the same sequence of pulse shapes. The observations appear to comply with the weakened dot confinement effect in the near off-center location of the dopant in comparison with the on-center location. The skirmish between this weakened confinement and the energy input also becomes less significant so far as $\left\langle R_{e x}\right\rangle$ is concerned. Thus, the extent of minimization has been substantially reduced. The observations at near off-center location thus categorically separate Gaussian shape pulse from saw-tooth pulse towards excitation. Thus, the role of pulse shape in modulating the excitation kinetics becomes more prominent in the near off-center dopant location in comparison with the on-center counterpart. More importantly, notwithstanding the shift of the dopant away from the dot confinement center, the particular Gaussian shape of the pulse seems to preserve the confinement strength intact.

The emergence of minima completely disappears at far off-center dopant location ( $r_{0}=70.71$ a.u.) for the Gaussian pulse. The Gaussian pulse $\left\langle R_{e x}\right\rangle$ exhibits a nearly steady character over a wide range of $n_{p}$ values. At this far off-center location, because of virtually insignificant confinement the excitation rate increases continually with $n_{p}$ without appearance of any minima and culminates in steady value. $\left\langle R_{e x}\right\rangle$ increases with $n_{p}$ for Gaussian pulse at low $n_{p}$ and finally undergoes saturation at $n_{p} \approx 6$. The saw-tooth pulse evinces minimization and saturation in $\left\langle R_{e x}\right\rangle$ at $n_{p} \approx 10$ and $n_{p}$ $\approx 14$, respectively. Thus, in the far off-center dopant location, it is the saw-tooth pulse that strives to preserve the confinement strength. However, owing to the heavily depleted dot-impurity overlap at this dopant location the saw-tooth pulse becomes partially successful in its endeavor. Table 1 summarizes the finer details of the effects of Gaussian and Saw-tooth pulse shapes on excitation kinetics for ready viewing. To be precise, table 1 demonstrates the $n_{p}$ values that correspond to minimization $\left(n_{p, \min }\right)$ and saturation $\left(n_{p, s a t}\right)$ of $\left(\left\langle R_{e x}\right\rangle\right)$ for the two types of pulse shapes. It also reveals the nature of the depth of minima.

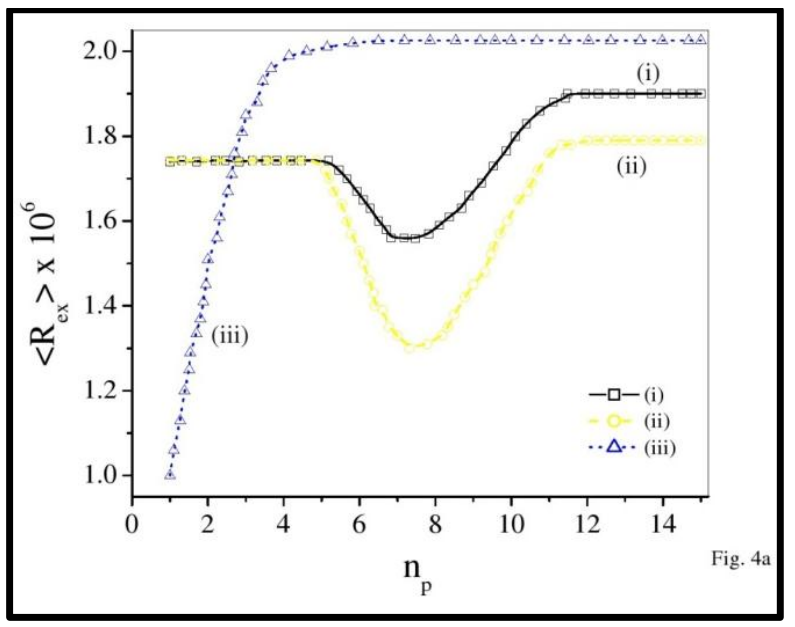

Fig. 4a. Plot of $\left\langle R_{e x}\right\rangle$ vs. $n_{p}$ for Gaussian pulse for different dopant locations: (i) on-center ( $r_{0}=0.0$ a.u.), (ii) near off-center $\left(r_{0}=28.28\right.$ a.u. $)$, and (iii) far offcenter $\left(r_{0}=70.71\right.$ a.u. $)$. 


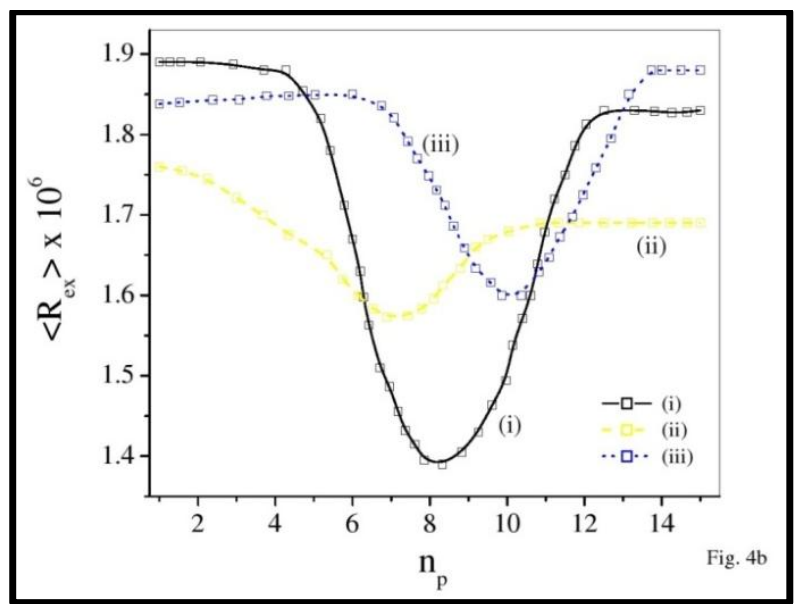

Fig. 4b. Plot of $\left\langle R_{e x}\right\rangle$ vs. $n_{p}$ for saw-tooth pulse for different dopant locations: (i) on-center $\left(r_{0}=0.0\right.$ a.u.), (ii) near off-center ( $r_{0}=28.28$ a.u.), and (iii) far offcenter $\left(r_{0}=70.71\right.$ a.u. $)$.

In the preceding sections we have given our thrust mainly on analyzing the role of $n_{p}$ on excitation kinetics at three different dopant locations. Now we turn our attention towards investigating the influence of dopant locations more exclusively on the excitation kinetics. For this purpose we have varied dopant coordinate $\left(r_{0}\right)$ over a range for fixed values of $n_{p}$. Earlier we came across that the excitation rate exhibits stable behavior as soon as $n_{p} \geq 15$ for both the pulse shapes. The stability guided us to fix $n_{p}$ equal to 15 and vary $r_{0}$ over a range. Fig. 5 depicts the $\left\langle R_{e x}\right\rangle$ vs. $r_{0}$ plots for both the pulse shapes at $n_{p}=15$. It needs to be realized now that the effect of gradual shift of dopant from on to off-center locations is not absolutely straightforward, rather it is self-contradictory. On one hand such shift weakens the strength of dot confinement thereby favoring excitation, but, on the other hand it also reduces dot-impurity repulsive interaction (because of decreasing overlap) and excitation is unfavored. Owing to these two opposite influences we expect appearance of maxima and minima in the excitation kinetics with a variation of dopant coordinate. The maxima or minima simply indicate a changeover in the dominance of one influence over its rival. The said shift of dopant mingled with typical shapes of both the pulses reveal diverse patterns of evolution of excitation kinetics which are worthmentioning. A close look at Fig. 5 exposes the said diversity elegantly; the saw-tooth pulse exhibits maxima at $r_{0}=40.50$ a.u. and the Gaussian pulse, starting from a rather low value, suddenly jumps to a high value at $r_{0}=$ 26.32 a.u. However, for both the pulses, at very far offcenter locations ( $r_{0} \geq 55$ a.u.) we envisage saturation in the excitation rate proclaiming ample balance between the rival factors that affect excitation.

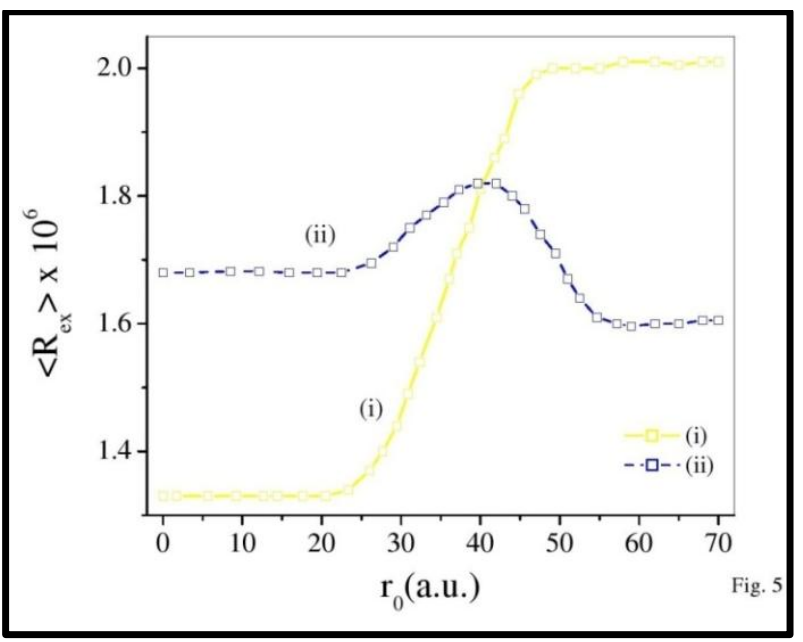

Fig. 5. Plot of $\left\langle R_{e x}\right\rangle$ vs. $r_{0}$ at $n_{p}=15$ for different pulse shapes: (i) Gaussian and (ii) saw-tooth.

Finally, we investigate the role of another two pulse parameters on the excitation kinetics. The parameters being the width factor $(k)$ for the Gaussian pulse and the height parameter $(a)$ for the saw-tooth pulse. Fig. 6a depicts the $\left\langle R_{e x}\right\rangle$ vs. $k$ plots for the Gaussian pulse at three distinct dopant locations viz. on-center $\left(r_{0}=0.0\right.$ a.u.), near off-center ( $r_{0}=28.28$ a.u.), and far off-center $\left(r_{0}=70.71\right.$ a.u.). An increase in $k$ value indicates a decrease in the width of the Gaussian pulse. From the plots, at all the three dopant locations the excitation rate is found to decrease consistently with decrease in the width of the pulse. However, at large $k$ value we envisage some sort of saturation in the excitation kinetics at all dopant locations. Thus, there is not much interplay between the width of the pulse and the dopant location. The scenario becomes widely different when we plot $\left\langle R_{e x}\right\rangle$ vs. $a$ for the saw-tooth pulse at above dopant locations [Fig. 6b]. The profiles are no longer similar at the three dopant locations as in the case of Gaussian pulse. Thus, the interplay between dopant location and the pulse height becomes indeed prominent for the saw-tooth pulse. At on center dopant location, $\left\langle R_{\theta X}\right\rangle$ decreases somewhat steadily with increase in $a$ before it undergoes saturation at $a \approx 1.5$ a.u. At near-off and far-off dopant locations, $\left\langle R_{e x}\right\rangle$ vs. $a$ plots exhibit maxima and minima, respectively. The location of maxima and minima being at $a \approx 0.65$ a.u. and $a \approx$ 0.76 a.u., respectively. It thus appears that, depending upon the dopant coordinate, a variation in the height of the saw-tooth pulse could bring about a twist in the relative dominance between the factors that promote and hinder excitation. At both near and far-off center locations, the excitation rate culminates in saturation as $a$ exceeds 1.4 a.u. and 1.2 a.u., respectively. 


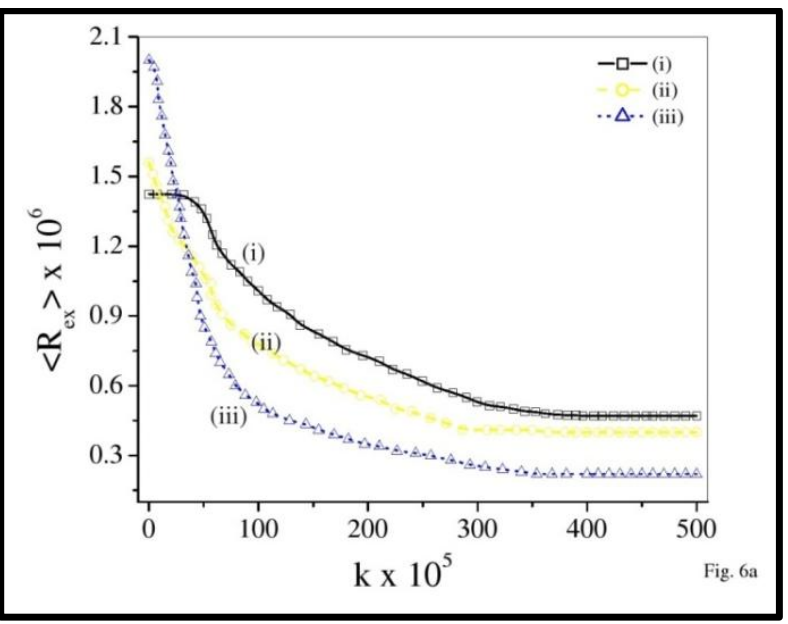

Fig. 6a. Plot of $\left\langle R_{e x}>\right.$ vs. $k$ for Gaussian pulse for different dopant locations: (i) on-center ( $r_{0}=0.0$ a.u.), (ii) near off-center $\left(r_{0}=28.28\right.$ a.u.), and (iii) far offcenter $\left(r_{0}=70.71\right.$ a.u. $)$.

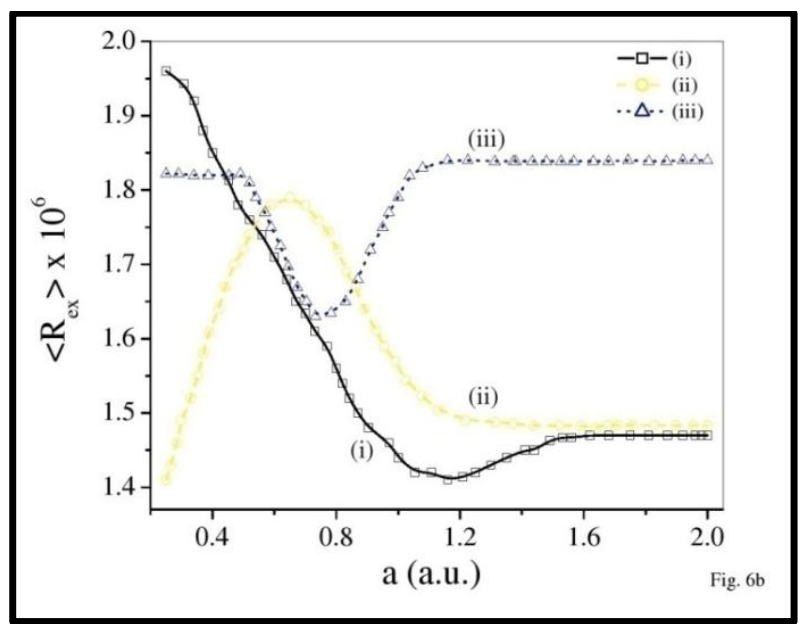

Fig. 6b. Plot of $\left\langle R_{e x}\right\rangle$ vs $a$ for saw-tooth pulse for different dopant locations: (i) on-center ( $r_{0}=0.0$ a.u.), (ii) near off-center $\left(r_{0}=28.28\right.$ a.u.), and (iii) far offcenter $\left(r_{0}=70.71\right.$ a.u. $)$.

\section{Conclusions}

The excitation kinetics of repulsive impurity doped quantum dots triggered by pulsed field of Gaussian and saw-tooth pulse shapes reveals noteworthy features. The dopant location and the number of pulses fed into the system, along with the typical shape of the pulse display prominent roles in modulating the excitation rate. Coupled with the dopant location, the width of the Gaussian pulse and the height of the saw-tooth pulse have also been found to influence the excitation kinetics. An on-center dopant location is endowed with a minimization of excitation rate at some particular $n_{p}$ values. When $n_{p}$ becomes quite large, we encounter saturation in the excitation rate for both pulse shapes. However, the specific role of pulse shape is revealed through the differences in the $n_{p}$ values corresponding to excitation minima, saturation and also through the varied depths of the excitation minima. The above features pertaining to typical shape of the pulse undergo modification with shift of the dopant to near off-center location and in the far off-center location the excitation minima disappears for Gaussian pulse. Moreover, analysis made to understand the exclusive role played by the dopant coordinate on excitation kinetics divulges pulse shape dependence to a great extent. The said dependence occurs owing to kind of changeover in the mutual dominance between the factors that favor and oppose the excitation process accompanying the dopant shift, and the changeover depends manifestly on pulse shape. At very far off-center locations the excitation kinetics reaches saturation for both the pulse shapes. A gradual shrinkage of the width of the Gaussian pulse causes a depletion in the excitation rate at all dopant locations. On the other hand, the result of steady increase in the height of the saw-tooth pulse is not at all straightforward. Such an increase modulates the excitation rate delicately depending upon the dopant location. We expect that the results obtained might reveal some important technological applications of doped quantum dot nanomaterials.

\section{Acknowledgements}

We thank Dr. Rahul Sharma, Department of Chemistry, St. Xavier's College, Kolkata, for his sincere cooperation.

\section{References}

[1] Poole Jr., C. P. and Owens, F. J. Introduction to Nanotechnology, Wiley, New York, 2003.

[2] Çakir, B.; Yakar, Y.; Özmen, A.; Özgür Sezer, M.; Şahin. M. Superlattices and Microstructures, 2010, 47, 556-566. http://dx.doi.org/10.1016/j.spmi.2009.12.002

[3] Movilla, J. L.; Planelles. J. Phys. Rev. B, 2005, 71, 075319- 075325.

http://dx.doi.org/10.1103/PhysRevB.71.075319

[4] Kelly, M. J. Low-dimensional Semiconductors, Oxford University Press, Oxford, 1995.

[5] Xie, W. Physica B, 2010, 405, 3436-3440. http://dx.doi.org/10.1016/j.physb.2010.05.019

[6] Xie, W. Superlattices. Microstructures, 2010, 48, 239-247. http://dx.doi.org/10.1016/j.spmi.2010.04.015 
[7] Yakar, Y.; Çakír, B.; Özmen, A. Optics Comm., 2010, 283, 1795-1800. http://dx.doi.org/10.1016/j.optcom.2009.12.027

[8] Peter, A. J. Phys. Lett. A, 2006, 355, 59-62. http://dx.doi.org/10.1016/j.physleta.2006.01.107

[9] Gulveren, B.; Atav, Ü.; Sahin, M.; Tomak, M. Physica E, 2005, 30, 143-149. http://dx.doi.org/10.1016/j.physe.2005.08.007

[10] Räsänen, E.; Könemann, J.; Puska, R. J.; Haug, M. J.; Nieminen, R. M. Phys. Rev. B, 2004, 70, 115308115313. http://dx.doi.org/10.1103/PhysRevB.70.115308

[11] Aichinger, M.; Chin, S. A.; Krotscheck, E.; Räsänen, E. Phys. Rev. B, 2006, 73, 195310-195317. http://dx.doi.org/10.1103/PhysRevB.73.195310

[12] Baskoutas, S.; Paspalakis, E.; Terzis, A. F. J. Phys:Cond. Mat., 2007, 19, 395024-395032. http://dx.doi.org/10.1088/0953-8984/19/39/395024

[13] Baskoutas, S.; Terzis, A. F.; Voutsinas, E. J. Comput. Theor. Nancosi, 2004, 1, 317-321.

[14] Nistor, S. V.; Stefan, M.; Nistor, L. C.; Goovaerts, E.; VanTendeloo, G. Phys. Rev. B, 2010, 81, 035336035341. http://dx.doi.org/10.1103/PhysRevB.81.035336

[15] Nistor, S. V.; Nistor, L. C.; Stefan, M.; Mateescua, C. D.; Birjega, R.; Solovieva, N.; Nikl, M. Superlattices. Microstructures, 2009, 46, 306-311. http://dx.doi.org/10.1016/j.spmi.2008.10.001

[16] Duque, C. A.; Porras-Montenegro, N.; Barticevic, Z.; Pacheco, M.; Oliveira, L. E. Microelectronic. J., 2005, 36, 231-233. http://dx.doi.org/10.1016/j.mejo.2005.04.001

[17] Duque, C. A.; Porras-Montenegro, N.; Barticevic, Z.; Pacheco, M.; Oliveira, L. E. J. Phys.: Condens. Matter, 2006, 18, 1877-1884. http://dx.doi.org/10.1088/0953-8984/18/6/005

[18] Paspalakis, E.; Terzis, A. F. Proceedings of the $5^{\text {th }}$ WSEAS International Conference on Microelectronics, Nanoelectronics, Optoelectronics, Prague, Czech Republic, March 12-14, (2006) (pp 44-49).

[19] Fountoulakis, A.; Terzis, A. F.; Paspalakis, E. J. Appl. Phys., 2009, 106, 074305-074312. http://dx.doi.org/10.1063/1.3232226

[20] Datta, N. K.; Ghosh, S.; Ghosh, M. J. Appl. Phys., 2010, 108, 104305-104311. http://dx.doi.org/10.1063/1.3510478

[21] Datta, N. K.; Ghosh, S.; Ghosh, M. Chem. Phys., 2010, 378, 66-72. http://dx.doi.org/10.1016/j.chemphys.2010.10.004
[22] Baskoutas, S.; Terzis, A. F. Eur. Phys. J. B, 2009, 69, 237-244. http://dx.doi.org/10.1140/epjb/e2009-00138-y

[23] Baskoutas, S.; Terzis, A. F. Physica E, 2008, 40, 1367-1370. http://dx.doi.org/10.1016/j.physe.2007.09.046

[24] Xie, W.; Xie, Q. Physica B, 2009, 404, 1625-1628. http://dx.doi.org/10.1016/j.physb.2009.01.037

[25] Boviatsis, J.; Voutsinas, E.; Fountoulakis, A. Superlattices and Microstructures, 2007, 41, 396399. http://dx.doi.org/10.1016/j.spmi.2007.03.019

[26] Halonen, V.; Hyvönen, P.; Pietiläinen, P.; Chakraborty, T. Phys. Rev. B, 1996, 53, 6971-6974. http://dx.doi.org/10.1103/PhysRevB.53.6971

[27] Halonen, V.; Pietiläinen, P.; Chakraborty, T. Europhys. Lett, 1996, 33, 337-382. http://dx.doi.org/10.1209/epl/11996-00350-5

[28] Jacak, L.; Hawrylak, P.; Wojos, A. Quantum Dots, Springer-Verlag, Berlin, 1998.

[29] Chakraborty, T. Quantum Dots-A Survey of the Properties of Artificial Atoms, Elsevier, Amsterdam, 1999.

[30] Adamowski, J.; Kwaśniowski, A.; Szafran, B. J. Phys:Cond. Mat., 2005, 17, 4489-4500. http://dx.doi.org/10.1088/0953-8984/17/28/008

[31] Bednarek, S.; Szafran, B.; Lis, K.; Adamowski, J. Phys. Rev. B, 2003, 68, 155333-155341. http://dx.doi.org/10.1103/PhysRevB.68.155333

[32] Szafran, B.; Bednarek, S.; Adamowski, J. Phys. Rev. B, 2001, 64, 125301- 125310. http://dx.doi.org/10.1103/PhysRevB.64.125301 